\title{
Collective dynamics in optomechanical arrays
}

\author{
Georg Heinrich, ${ }^{1}$ Max Ludwig, ${ }^{1}$ Jiang Qian, ${ }^{2}$ Björn Kubala, ${ }^{1}$ and Florian Marquardt ${ }^{1,3}$ \\ ${ }^{1}$ Institute for Theoretical Physics, Universität Erlangen-Nürnberg, Staudtstr. 7, 91058 Erlangen, Germany \\ ${ }^{2}$ Department of Physics, Center for NanoScience, \\ and Arnold Sommerfeld Center for Theoretical Physics, \\ Ludwig-Maximilians-Universität München, Theresienstr. 37, D-80333 München, Germany \\ ${ }^{3}$ Max Planck Institute for the Science of Light, Günter-Scharowsky-Straße 1/Bau 24, 91058 Erlangen, Germany
}

The emerging field of optomechanics seeks to explore the interaction between nanomechanics and light (see [1] for a recent review). Rapid progress in laser cooling of nanomechanical oscillators [2, 3] promises new fundamental tests of quantum mechanics [4], while applications benefit from ultrasensitive detection of displacements, masses and forces [5-7]. Recently, the exciting concept of optomechanical crystals has been introduced [8-10], where defects in photonic crystal structures are used to generate both localized optical and mechanical modes that interact with each other. For instance, this opens the prospect of integrated optomechanical circuits combining several functions on a single chip (see also [11, 12]). Here we start exploring the collective dynamics of arrays consisting of many coupled optomechanical cells (Fig. 1 1 a,b). We show that such "optomechanical arrays" can display synchronization and that they can be described by a modified Kuramoto model that allows to explain and predict most of the features that will be observable in future experiments.

The crucial ingredient of any optomechanical system is an optical mode (OM) whose frequency shifts in response to a mechanical displacement: $\delta \omega_{\text {opt }}=-G x$. This coupling, vice versa, gives rise to a radiation force, $F=\hbar G|\alpha|^{2}$, where $|\alpha|^{2}$ is the number of photons circulating inside the laser-driven OM. For a laser red-detuned from the OM $\left(\Delta=\omega_{\text {Laser }}-\omega_{\text {opt }}<0\right)$, dynamical backaction effects induced by the finite photon decay time $\kappa^{-1}$ lead to cooling of the mechanical motion. In contrast, for blue detuning $(\Delta>0)$, anti-damping results. Once this overcomes the internal mechanical friction, a Hopf bifurcation towards a regime of self-induced mechanical oscillations takes place (Fig. 11) [13 18. While the mechanical amplitude $A$ is fixed, the oscillation phase $\varphi$ is undetermined. Therefore, it is susceptible to external perturbations. In particular, as we will see, it may lock to external forces or to other optomechanical oscillators.

Synchronization has first been discovered by Huygens and is now recognized as an important feature of collective nonequilibrium behavior in fields ranging from physics over chemistry to biology and neuroscience [19], with applications in signal processing and stabilization

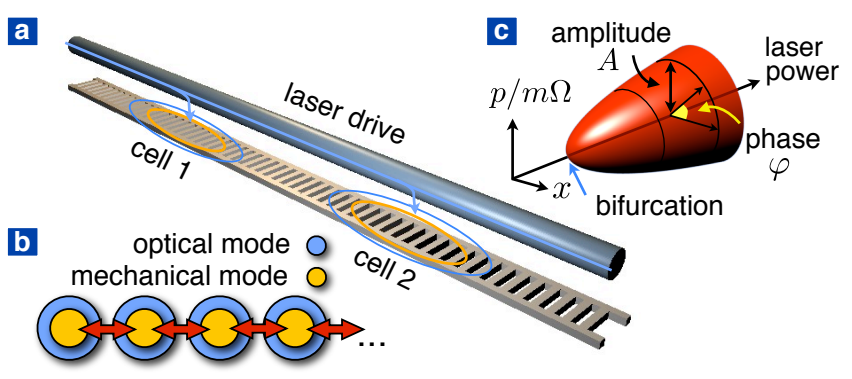

FIG. 1. Optomechanical crystals may be used to build arrays with several localized optical and mechanical modes. (a) Potential setup fabricated as a periodically patterned, freestanding dielectric beam on a chip with laser drive via tapered fibre as in 8, 9]. (b) Schematic array of mechanically coupled optomechanical cells. (c) For a single cell, at sufficient laser drive power, there is a Hopf bifurcation towards self-induced oscillations with an undetermined phase $\varphi$.

of oscillations. A paradigmatic, widely studied model for synchronization was introduced by Kuramoto [20]. For two oscillators, his phase evolution equation reads $\dot{\varphi}_{1}=\Omega_{1}+K \sin \left(\varphi_{2}-\varphi_{1}\right)$, and likewise $\dot{\varphi}_{2}$. One finds synchronization $\left(\dot{\varphi}_{1}=\dot{\varphi}_{2}\right)$ if the coupling $K$ exceeds the threshold $K_{c}=\left|\Omega_{2}-\Omega_{1}\right| / 2$, and the phase lag $\delta \varphi=\varphi_{2}-\varphi_{1}$ vanishes for large $K$ according to $\sin (\delta \varphi)=\left(\Omega_{2}-\Omega_{1}\right) / 2 K$. For the globally coupled, meanfield type version of infinitely many oscillators, there is a phase transition towards synchronization beyond some threshold $K_{c}$ that depends on the frequency distribution 21. In many examples the Kuramoto model is found as a generic, reduced description of the phase dynamics. Nevertheless, for any specific system, it remains to be seen whether this model (or possibly a structurally similar variant thereof) applies at all, and how the coupling $K$ is connected to microscopic parameters 22 24]. We now turn to this question in the case of optomechanical oscillators.

A single optomechanical cell consists of a mechanical mode (displacement $x$ ) coupled to a laser-driven OM (light amplitude $\alpha$ ):

$$
\begin{aligned}
m \ddot{x} & =-m \Omega^{2} x-m \Gamma \dot{x}+\hbar G|\alpha|^{2} \\
\dot{\alpha} & =\left[i(\Delta+G x)-\frac{\kappa}{2}\right] \alpha+\frac{\kappa}{2} \alpha_{\max }
\end{aligned}
$$

Here $\Omega$ is the mechanical frequency, $\Gamma$ the intrinsic damping, $G$ the optomechanical frequency pull per displace- 
ment, and $\alpha_{\max }$ is the maximum light-field amplitude achieved at resonance (set by the laser drive).

Near the Hopf bifurcation (Fig. 1;), we can capture the essential dynamics by eliminating the light field 16 and switching to the phase- and amplitude-dynamics of the resulting Hopf oscillator:

$$
\begin{aligned}
& \dot{\varphi}=-\Omega+\frac{F(t)}{m \Omega A} \cos (\varphi) \\
& \dot{A}=-\gamma(A-\bar{A})+\frac{F(t)}{m \Omega} \sin (\varphi) .
\end{aligned}
$$

Here $\bar{A}$ is the steady-state amplitude, and $\gamma$ is the rate at which perturbations will relax back to $\bar{A}$. Both depend on the microscopic optomechanical parameters, such as laser detuning and laser drive power, and both vanish at the bifurcation threshold (see methods section). Moreover, we have introduced an external force $F(t)$ (as added to Eq. (2))

We start our discussion by considering phase-locking to an external force $F(t)=F_{0} \sin \left(\omega_{F} t\right)$. To this end we time-average Eq. (3), keeping only the slow dynamics, under the assumption $\omega_{F} \approx \Omega$. This results in

$$
\delta \dot{\varphi}=-\delta \Omega+K_{F} \sin (\delta \varphi),
$$

where $\delta \varphi=\varphi(t)+\omega_{F} t, \delta \Omega=\Omega-\omega_{F}$, and $K_{F}=$ $F_{0} / 2 m \Omega \bar{A}$. Eq. 5 is a special case of the Kuramoto equation. Direct numerical simulation confirms the good agreement between the microscopic optomechanical dynamics and the simplified descriptions via Eqs. (34) and (5). In Fig. 2 we show $\sin \delta \varphi(t)$ and its time-average $\langle\sin \delta \varphi(t)\rangle$, with the phase $\varphi$ being extracted from the complex amplitude of motion, $\beta \equiv x+i \dot{x} / \Omega$. Phaselocking sets in when $\delta \dot{\varphi}=0$ has a solution, i.e. for $|\delta \Omega| \leq K_{F}$, resulting in an "Arnold tongue" (see Fig. $2 \mathrm{~d}$ ).

We now turn to the dynamics of coupled cells, each of which is described by Eqs. (1, 2). To these equations, we add a mechanical coupling, set by a spring constant $k$ : $m \ddot{x}_{1}=\ldots+k\left(x_{2}-x_{1}\right)$. In the Hopf model, this yields a force $F_{1}=k A_{2} \cos \left(\varphi_{2}\right)$ on the first oscillator (and vice versa). The case of optical coupling will be mentioned further below.

In order to arrive at the time-averaged dynamics for the phase difference, $\delta \varphi=\varphi_{2}-\varphi_{1}$, it is necessary to go further than before, keeping $A(t)=\bar{A}+\delta A(t)$ in the phase equation, and eliminating the amplitude dynamics to lowest order (see methods for the derivation; and 25. 26] for further examples where the amplitude dynamics is crucial). Then, we arrive at an effective Kuramoto-type model for coupled optomechanical Hopf oscillators:

$$
\delta \dot{\varphi}=-\delta \Omega-C \cos (\delta \varphi)-K \sin (2 \delta \varphi) .
$$

In contrast to the standard Kuramoto model, $2 \delta \varphi$ appears, which will lead to both in-phase and anti-phase
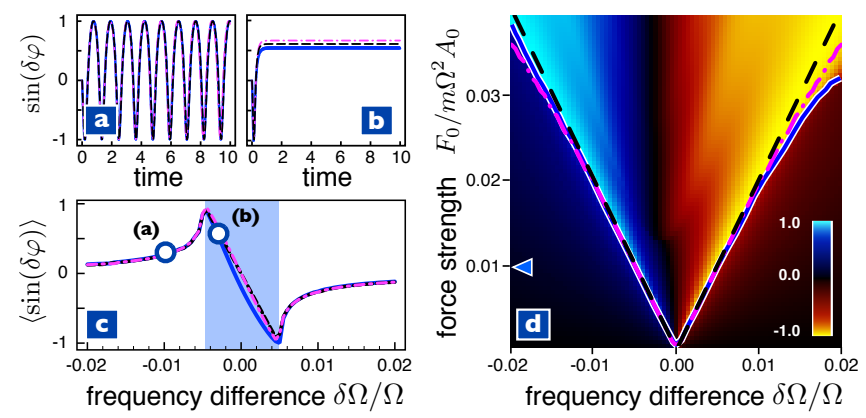

FIG. 2. Phase-locking of an optomechanical cell to an external force. (a,b) Phase lag $\delta \varphi$ between oscillations and force, displayed via $\sin \delta \varphi$, outside (a) and inside (b) the phaselocked regime (colored in (c)). Time is plotted in units of $2 \pi / \delta \Omega$. (c) Plot of $\langle\sin \delta \varphi\rangle$ as a function of frequency difference $\delta \Omega$, at the force strength indicated by the blue triangle in (d), comparing optomechanics (blue, solid) against the Hopf model (Eqs. (34); magenta, dash-dot) and the Kuramoto model (Eq. (5); black, dash). (d) $\langle\sin \delta \varphi\rangle$ in the force vs. frequency difference plane, showing the Arnold tongue. Deviations between the different models set in at high forces. (Lines indicate the transition towards phase-locking, styles as in (c)). (Microscopic cell parameters: $\Delta / \Omega=1, \kappa / \Omega=1$, $\left.\Gamma / \Omega=0.01, \hbar G^{2} \alpha_{\max }^{2} / m \Omega^{3}=0.36\right)$.

synchronization. This corresponds to two distinct minima in the effective potential that can be used to rewrite Eq. 60: $\delta \dot{\varphi}=-U^{\prime}(\delta \varphi)$. The coupling $K=k^{2} / 2 m^{2} \Omega^{2} \gamma$ diverges near the bifurcation, where $\gamma \rightarrow 0$. In the following we focus on the case of nearly identical cells where the coupling $C$ can be neglected; $C / \delta \Omega=k / 2 m \Omega^{2} \ll 1$.

To test whether these features are observed in the full optomechanical system, we directly simulate the motion and increase the coupling $k$ for a fixed frequency difference $\delta \Omega=\Omega_{2}-\Omega_{1}$. The results are displayed in Fig. 33(ac). Beyond a threshold $k_{c}$, the frequencies and the phases lock, indicated by a kink in $\langle\sin \delta \varphi\rangle$. As the coupling increases further, the phases are pulled towards each other even more, so $|\delta \varphi|$ decreases. Thus, coupled optomechanical systems do indeed exhibit synchronization. As predicted, there is both synchronization towards $\delta \varphi \rightarrow 0$ and $\delta \varphi \rightarrow \pi$.

The dependence of the threshold $k_{c}$ on the frequency difference $\delta \Omega$ is shown in Fig. $3 \mathrm{~d}$. The observed behavior $k_{c} \sim \sqrt{\delta \Omega}$ at small $\delta \Omega$ is correctly reproduced by the generalized Kuramoto model, Eq. (6). For $\delta \Omega>\gamma$ deviations occur via terms of higher order in $\delta \Omega / \gamma$, starting with $-(\delta \Omega / \gamma) K \cos (2 \delta \varphi)$ in Eq. (6). These produce a linear slope $k_{c} \propto \delta \Omega$, see Fig. 33.

In terms of experimental realization, optomechanical crystals [8, 9] offer a novel promising way to build arrays of optomechanical cells. They are fabricated as free-standing photonic crystal beams (Fig. 1 1). Variations of the $\mu m$-scale lattice spacing produce both localized optical and vibrational modes. The strong confine- 

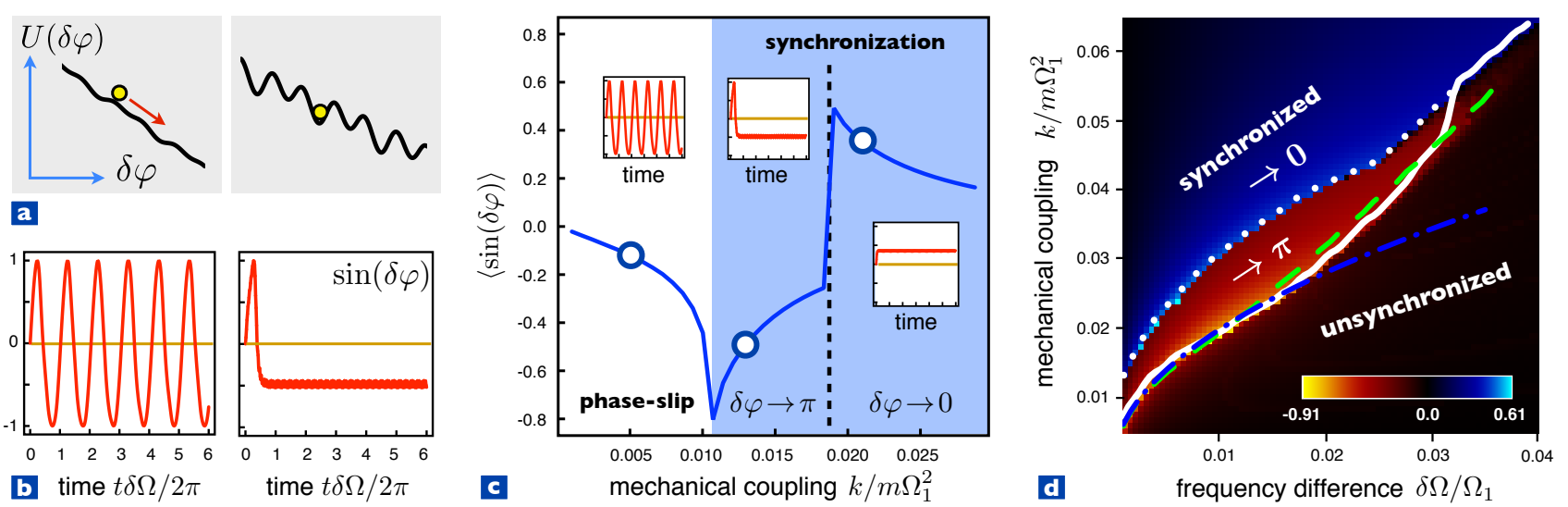

FIG. 3. Phase-locking for two coupled optomechanical cells. (a) The phase particle in the effective Kuramoto potential $U(\delta \varphi)$, in the de-synchronized and phase-locked regime (left/right). (b) Time-evolution of $\sin (\delta \varphi(t))$. (c) When the mechanical coupling $k$ exceeds a threshold, the phase difference $\delta \varphi$ between the oscillations locks in spite of different bare mechanical frequencies, here $\delta \Omega=0.003 \Omega_{1}$. Both in-phase and anti-phase regimes are observed. (d) Phase lag, expressed via $\langle\sin (\delta \varphi)\rangle$, in the plane coupling $k$ vs. frequency difference $\delta \Omega$, including a comparison of the critical coupling $k_{c}$ (white, solid) with the one from a Hopf model with one fit parameter (green, dash) and the generalized Kuramoto model (blue, dash-dot). (Cell parameters as in Fig. 2).

ment leads to extremely large optomechanical couplings, on the order of $G \sim 100 \mathrm{GHz} / \mathrm{nm}$. Typical parameters, that we use in our simulations for experimentally realistic results, are $G=100 \mathrm{GHz} / \mathrm{nm}$, mechanical frequency $\Omega=1 \mathrm{GHz}$, mass $m=100 \mathrm{fg}$, mechanical quality factor $Q_{M}=\Omega / \Gamma=100$, cavity decay rate $\kappa=1 \mathrm{GHz}$ and laser input powers such that the circulating photon number $\left|\alpha_{\max }\right|^{2} \gtrsim 100$.

To consider optomechanical arrays like in Fig. 11a, we use finite element methods (FEM) to simulate two identical cells arranged on the same beam (Fig. 4a,b). The optical and vibrational couplings mediated by the beam can be deduced from the splitting between the resulting symmetric/antisymmetric modes. The results shown in Fig. 4k,d validate the parameters considered above and indicate mechanical couplings $k / m \Omega^{2} \lesssim 0.01$. Due to the relatively strong optical coupling $(\sim \mathrm{THz})$, distinct OMs in the individual cells can only be achieved by patterning them to have frequencies sufficiently different to prevent hybridization (Fig, 4 4 ). This requires different laser colors to address each cell.

In experiments, a convenient observable would be the RF frequency spectrum of the light intensity emanating from the cells, $|\alpha|^{2}(\omega)$. We first show the spectrum as a function of frequency difference $\delta \Omega$ for two mechanically coupled cells, driven independently (Fig. 5a). Frequency locking is observed in an interval around $\delta \Omega=0$. Experimentally, the mechanical frequencies can be tuned via the "optical spring effect" [8].

The most easily tunable parameter is the laser drive power $\left(\propto \alpha_{\max }^{2}\right)$. Synchronization sets in right at the Hopf bifurcation. For two cells (Fig. 5p), we recover the regimes of in-phase and anti-phase synchronization. They differ in the synchronization frequency, $\bar{\Omega}(\pi)-$

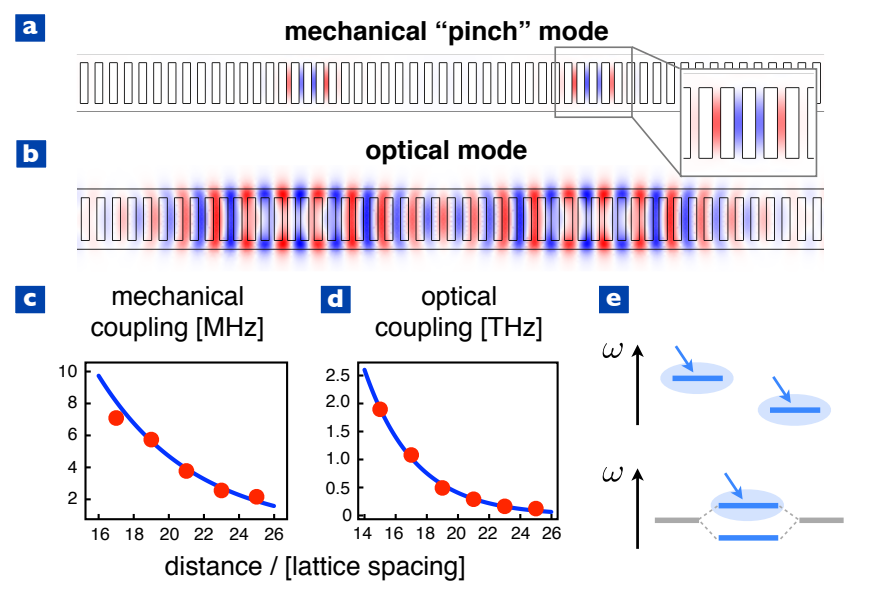

FIG. 4. FEM simulation for two coupled cells localized on the same beam in an optomechanical crystal setup (cf. Fig. 117). (a) Horizontal displacement of vibrational "pinch" modes. (b) Transverse electric fields of optical modes. (c,d) The intercell couplings decay exponentially as a function of distance. (e) Blue-detuned lasers could illuminate two distinct optical modes (top) or a single hybridized "molecular" mode. (Geometrical parameters see methods section).

$\bar{\Omega}(0)=k / m \Omega$. At higher drive, we find a transition towards de-synchronization. This remarkable behavior can be explained from our analytical results. We know that $\gamma$ increases away from the Hopf bifurcation (i.e. for higher drive), leading to a concomitant decrease in the effective Kuramoto coupling $K \propto 1 / \gamma$, and finally a loss of synchronization. Near the transition, the frequencies fan out (as $\delta \Omega_{\text {eff }} \propto \sqrt{\alpha_{\max }-\alpha_{c}}$ ). The multitude of peaks is produced due to nonlinear mixing. In some regimes, 

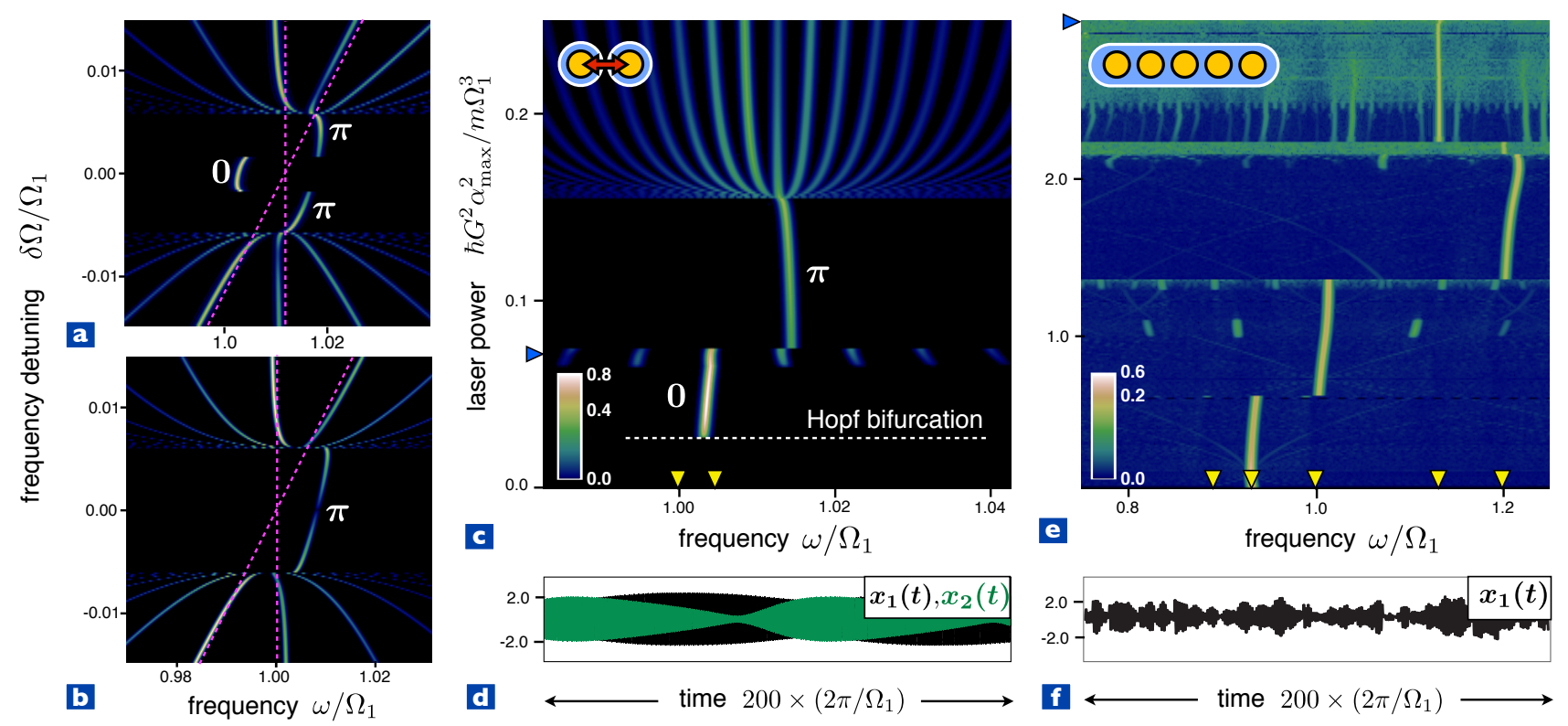

FIG. 5. Mechanical frequency spectra of optomechanical arrays. (a,b) Frequency locking upon changing the detuning $\delta \Omega=\Omega_{2}-$ $\Omega_{1}$ between the mechanical frequencies (magenta, dashed) of two independently driven, mechanically coupled optomechanical cells $\left(k / m \Omega_{1}^{2}=0.015\right)$. (a) Spectrum $I(\omega)$ of intensity fluctuations, $I(t)=\left|\alpha_{1}(t)\right|^{2}+\left|\alpha_{2}(t)\right|^{2}$, from an optomechanical model. (b) Spectrum of $x_{1}+x_{2}$ in the Hopf model. (c) Spectrum vs. laser input power for two independently driven, mechanically coupled cells $\left(k / m \Omega_{1}^{2}=0.01, \delta \Omega=0.005 \Omega_{1}\right)$. (d) Example of trajectories $x_{i}(t)$, in units of $\kappa / G$, displaying strong amplitude modulation, not described by the Kuramoto model (at a power indicated by the blue triangle in (c)). (e) Spectrum $I(\omega)$ vs. laser input power for an array of five mechanical modes $(k=0)$, coupled to a common optical mode. At large drive, a regime of chaotic motion is entered. Due to the presence of multiple attractors, the regimes observed in such a diagram may depend on how the parameters are swept. (f) Example of a trajectory in the chaotic regime (blue triangle in (e)). (color scale in all plots indicates $|I(\omega)|$ in units of the peak height at $\omega=0$ for a system at rest; $\delta$ peaks are broadened for clarity; cell parameters are $\Delta_{i}=\kappa_{i}=100 \Gamma_{i}=\Omega_{1}$, as in Figs. 233, yellow triangles indicate the bare mechanical frequencies $\Omega_{i}$ )

the Kuramoto model fails (Fig. 5d).

For large arrays, it will be most practical to have identical OMs that combine into extended 'molecular' modes, one of which is then driven by a single laser via an evanescently coupled tapered fibre (Fig. 13, Fig. 4 ). For efficient excitation of self-induced oscillations, one has to ensure that the detuning $\Delta$ is equal to all the mechanical frequencies $\Omega_{j}$ in the array, to within $\left|\Delta-\Omega_{j}\right|<\kappa$. For arrays of reasonable size, the splittings between adjacent optical molecular modes will be more than $10^{2}$ times larger than $\kappa$, such that we can ignore all but one OM. This setup then leads to a global coupling of many nanoresonators to a single OM, such that

$$
\dot{\alpha}=\left[i\left(\Delta+\sum_{j} G_{j} x_{j}\right)-\frac{\kappa}{2}\right] \alpha+\frac{\kappa}{2} \alpha_{\max },
$$

and the force on each resonator is given by $-\hbar G_{j}|\alpha|^{2}$. This setup comes close to realizing the all-to-all coupling most often investigated in the literature on the Kuramoto model.

For illustration, we chose $N=5$ cells (Fig. 5e). As before, we find synchronization regimes. In addition, at higher drive, a transition into chaos takes place. Analyzing the time-evolution in more detail, we observe transient fluctuations in amplitude and phase, with a strong sensitivity on changes in initial conditions (Fig. 5f). Note that in a single optomechanical cell one may also find chaotic behavior [14, but for far larger driving strengths.

Optomechanical arrays open up a new domain to study collective oscillator dynamics, with room-temperature operation in integrated nanofabricated circuits and with novel possibilities for readout and control, complementing existing research on Josephson arrays [22, laser arrays [23] and other nanomechanical structures [24, 27]. Recent experiments on 2D optomechanical crystals 28] could form the basis for investigating collective dynamics in $2 \mathrm{D}$ settings with various coupling schemes. Applications in signal processing may benefit from phase noise suppression via synchronization 29. Variations of the optomechanical arrays investigated here may also be realized in other designs based on existing setups, like multiple membranes in an optical cavity [30] or arrays of toroidal microcavities [2, 5. 


\section{Acknowledgements}

We thank O. Painter, H. Tang and J. Parpia for fruitful discussions, and GIF and DFG (Emmy-Noether program, NIM) for funding.

\section{Methods}

The dynamics of a single optomechanical cell, Eqs. 11, 2, in the self-induced oscillation regime can be mapped to a Hopf model close to the Hopf bifurcation. This model is described by the steady state amplitude $\bar{A}$ and amplitude decay rate $\gamma$ (see Eqs. (3, 4)). The dependence of $\gamma, \bar{A}$ on the microscopic parameters can be deduced by expanding the average mechanical power input provided by the radiation pressure force, $\hbar G\left\langle|\alpha|^{2} \dot{x}\right\rangle$ (see Eq. (1)), in terms of $a=G \bar{A}$; $\hbar G\left\langle|\alpha|^{2} \dot{x}\right\rangle=\left(\hbar \alpha_{\max }^{2} \pi_{2}\right) a^{2}+\left(\hbar \alpha_{\max }^{2} \pi_{4} / \Omega^{2}\right) a^{4}+O\left(a^{6}\right)$. This yields

$$
\gamma=2 \mathcal{P} \Omega \pi_{2}-\Gamma
$$

and

$$
(G \bar{A} / \kappa)^{2}=\gamma \Omega /\left(-2 \pi_{4} \mathcal{P} \kappa^{2}\right)
$$

where the dimensionless coefficients $\pi_{2}(\Delta / \Omega, \kappa / \Omega)$ and $\pi_{4}(\Delta / \Omega, \kappa / \Omega)$ only depend on the rescaled detuning and cavity decay rate. $\mathcal{P}=\hbar G^{2} \alpha_{\max }^{2} / m \Omega^{3}$ is the rescaled laser input power. Numerically, the Hopf parameters may be found (even away from threshold) by simulating the exponential relaxation of the cell's oscillation amplitude towards $\bar{A}$, after a small instantaneous perturbation of the steady-state dynamics. In general, to compare optomechanics to results from Hopf (e.g. Fig. 2), the optical spring effect also needs to be considered.

Two mechanically coupled optomechanical cells are modeled as distinct Hopf oscillators with phase dynamics $\varphi_{1}(t), \varphi_{2}(t)$ and amplitude dynamics $A_{1}(t), A_{2}(t)$ according to Eqs. (3) and (4). The coupling forces read $F_{1}=k A_{2} \cos \left(\varphi_{2}\right), F_{2}=k A_{1} \cos \left(\varphi_{1}\right)$. With $A_{i}(t)=\bar{A}_{i}+\delta A_{i}(t)$, the solution for the amplitude dynamics is $\delta A_{i}(t)=\int_{-\infty}^{t} e^{-\gamma\left(t-t^{\prime}\right)} \tilde{f}_{i}\left(t^{\prime}\right) d t^{\prime}$ where $\tilde{f}_{1}(t)=$ $\frac{k\left(\bar{A}_{2}+\delta A_{2}(t)\right)}{m_{1} \Omega_{1}} \cos \varphi_{2}(t) \sin \varphi_{1}(t)$ and likewise for $\tilde{f}_{2}(t)$. In the following, we consider small couplings, where $\delta A_{i} \ll$ $\bar{A}_{i}$. Then $\delta A_{1}$ (and likewise $\delta A_{2}$ ) is found to be

$$
\begin{aligned}
\frac{\delta A_{1}(t)}{\bar{A}_{1}}= & \frac{k}{2 m_{1} \Omega_{1}} \times \\
& \operatorname{Im}\left(\frac{e^{i\left(\varphi_{2}(t)+\varphi_{1}(t)\right)}}{\gamma-i\left(\Omega_{2}+\Omega_{1}\right)}-\frac{e^{i\left(\varphi_{2}(t)-\varphi_{1}(t)\right)}}{\gamma-i\left(\Omega_{2}-\Omega_{1}\right)}\right) .
\end{aligned}
$$

Thus we can eliminate the amplitude dynamics to lowest order from the phase equations, by expanding
$A_{2}(t) / A_{1}(t) \approx \bar{A}_{2} / \bar{A}_{1}+\delta A_{2} / \bar{A}_{1}-\bar{A}_{2} \delta A_{1} / \bar{A}_{1}$ in the following equation (likewise for $\varphi_{2}$ ):

$$
\dot{\varphi}_{1}=-\Omega_{1}+\frac{k}{m_{1} \Omega_{1}}\left(\frac{A_{2}}{A_{1}}\right) \cos \left(\varphi_{2}\right) \cos \left(\varphi_{1}\right) .
$$

We now perform a time average, keeping only the slow dynamics near frequencies 0 and $\pm\left|\Omega_{2}-\Omega_{1}\right|$. This leads to the stated result for the effective $\mathrm{Ku}$ ramoto model, Eq. [6], after setting $\delta \varphi=\varphi_{2}-$ $\varphi_{1}$. The coupling constants are given by $C=$ $(k / 2)\left(\bar{A}_{2} / m_{1} \Omega_{1} \bar{A}_{1}-\bar{A}_{1} / m_{2} \Omega_{2} \bar{A}_{2}\right)$ and $K=(1+$ $\left.\left(\xi_{1} / \xi_{2}+\xi_{2} / \xi_{1}\right) / 2\right) k^{2} / 4 m_{1} \Omega_{1} m_{2} \Omega_{2}$, where $\xi_{j}=m_{j} \Omega_{j} \bar{A}_{j}^{2}$.

The optomechanical simulation in Fig. 3 shows results for experimentally realistic microscopic parameters using an input power well above the bifurcation threshold. This allows to observe the essential features predicted from Hopf and the effective Kuramoto-type model in an appropriate range of frequency detuning $\delta \Omega$. However, to achieve quantitative agreement of the Hopf model in Fig. 3, its parameter $\gamma$ has to be treated as an adjustable parameter (here $\gamma=0.02 \Omega$ ). Each simulation initially starts with a system at rest and considers an instantaneous switch-on of the laser input power. Whether the system synchronizes towards $\delta \varphi \rightarrow 0$ or $\delta \varphi \rightarrow \pi$ also depends on the initial conditions.

In principle, an amplitude dependence of the mechanical frequency, $\Omega(A) \simeq \Omega(\bar{A})+(\partial \Omega / \partial A) \delta A$ can yield additional terms and an alternative synchronization mechanism for two Hopf oscillators. However, numerical simulations verified that for optomechanical cells this aspect can be neglected.

For the finite-element simulation in Fig. 4 the unit cell in the periodic part is a $1,396 \mathrm{~nm}$-wide, $362 \mathrm{~nm}$-long rectangle with a co-centric rectangular hole of $992 \mathrm{~nm}$ width and 190nm length. The thickness of the beam is $220 \mathrm{~nm}$. The isotropic Young's modulus of $168.5 \mathrm{GPa}$ and the refractive index is 3.493. Each defect is 15 units in length, symmetric across the 8 th(central) cell. The lattice constants vary linearly from $362 \mathrm{~nm}$ at the edge to $307.7 \mathrm{~nm}$ at the center. The holes in the defects stay co-centric with the unit cell and remain constant in size. For a single cell these parameters have been reported in Ref. [9].

[1] Marquardt, F. \& Girvin, S. M. Optomechanics. Physics 2, 40 (2009).

[2] Schliesser, A., Arcizet, O., Riviere, R., Anetsberger, G. \& Kippenberg, T. J. Resolved-sideband cooling and position measurement of a micromechanical oscillator close to the Heisenberg uncertainty limit. Nat. Phys. 5, 509-514 (2009).

[3] Gröblacher, S. et al. Demonstration of an ultracold micro-optomechanical oscillator in a cryogenic cavity. Nat. Phys. 5, 485-488 (2009).

[4] Marshall, W., Simon, C., Penrose, R. \& Bouwmeester, 
D. Towards quantum superpositions of a mirror. Phys. Rev. Lett. 91, 130401 (2003).

[5] Anetsberger, G. et al. Near-field cavity optomechanics with nanomechanical oscillators. Nat. Phys. 5, 909-914 (2009).

[6] Verlot, P., Tavernarakis, A., Briant, T., Cohadon, P.-F. \& Heidmann, A. Scheme to probe optomechanical correlations between two optical beams down to the quantum level. Phys. Rev. Lett. 102, 103601 (2009).

[7] Teufel, J. D., Donner, T., Castellanos-Beltran, M. A., Harlow, J. W. \& Lehnert, K. W. Nanomechanical motion measured with an imprecision below that at the standard quantum limit. Nat. Nano. 4, 820-823 (2009).

[8] Eichenfield, M., Camacho, R., Chan, J., Vahala, K. J. \& Painter, O. A picogram- and nanometre-scale photoniccrystal optomechanical cavity. Nature 459, 550-555 (2009).

[9] Eichenfield, M., Chan, J., Camacho, R. M., Vahala, K. J. \& Painter, O. Optomechanical crystals. Nature 462, 7882 (2009).

[10] Chang, D., Safavi-Naeini, A. H., Hafezi, M. \& Painter, O. Slowing and stopping light using an optomechanical crystal array. arXiv: 1006.3829 (2010).

[11] Li, M. et al. Harnessing optical forces in integrated photonic circuits. Nature 456, 480-484 (2008).

[12] Li, M., Pernice, W. H. P. \& Tang, H. X. Tunable bipolar optical interactions between guided lightwaves. Nat. Photon. 3, 464-468 (2009).

[13] Höhberger, C. \& Karrai, K. Self-oscillation of micromechanical resonators. Nanotechnology 2004, Proceedings of the 4th IEEE conference on nanotechnology 419 (2004).

[14] Carmon, T., Rokhsari, H., Yang, L., Kippenberg, T. J. \& Vahala, K. J. Temporal behavior of radiation-pressureinduced vibrations of an optical microcavity phonon mode. Phys. Rev. Lett. 94, 223902 (2005).

[15] Kippenberg, T. J., Rokhsari, H., Carmon, T., Scherer, A. \& Vahala, K. J. Analysis of radiation-pressure induced mechanical oscillation of an optical microcavity. Phys. Rev. Lett. 95, 033901 (2005).

[16] Marquardt, F., Harris, J. G. E. \& Girvin, S. M. Dynamical multistability induced by radiation pressure in high-finesse micromechanical optical cavities. Phys. Rev. Lett. 96, 103901 (2006).
[17] Ludwig, M., Kubala, B. \& Marquardt, F. The optomechanical instability in the quantum regime. New J. Phys. 10, 095013 (2008).

[18] Metzger, C. et al. Self-induced oscillations in an optomechanical system driven by bolometric backaction. Phys. Rev. Lett. 101, 133903 (2008).

[19] Pikovsky, A., Rosenblum, M. \& Kurths, J. Synchronization: A Universal Concept in Nonlinear Sciences (Cambridge University Press, 2001).

[20] Kuramoto, Y. Self-entrainment of a population of coupled nonlinear oscillators. In International Symposium on Mathematical Problems in Theoretical Physics, vol. 39, 420-422 (Springer, 1975).

[21] Acebrón, J. A., Bonilla, L. L., Pérez Vicente, C. J., Ritort, F. \& Spigler, R. The Kuramoto model: A simple paradigm for synchronization phenomena. Rev. Mod. Phys. 77, 137-185 (2005).

[22] Wiesenfeld, K., Colet, P. \& Strogatz, S. H. Synchronization transitions in a disordered Josephson series array. Phys. Rev. Lett. 76, 404-407 (1996).

[23] Kozyreff, G., Vladimirov, A. G. \& Mandel, P. Global coupling with time delay in an array of semiconductor lasers. Phys. Rev. Lett. 85, 3809-3812 (2000).

[24] Cross, M. C., Zumdieck, A., Lifshitz, R. \& Rogers, J. L. Synchronization by nonlinear frequency pulling. Phys. Rev. Lett. 93, 224101 (2004).

[25] Aronson, D., Ermentrout, G. \& Kopell, N. Amplitude response of coupled oscillators. Physica D 41, $403-449$ (1990).

[26] Matthews, P. C., Mirollo, R. E. \& Strogatz, S. H. Dynamics of a large system of coupled nonlinear oscillators. Physica D 52, 293 - 331 (1991).

[27] Zalalutdinov, M. et al. Frequency entrainment for micromechanical oscillator. Appl. Phys. Lett. 83, 3281-3283 (2003).

[28] Safavi-Naeini, A. H., Mayer Alegre, T. P., Winger, M. \& Painter, O. Optomechanics in an ultrahigh-Q slotted 2D photonic crystal cavity. arXiv: 1006.3964 (2010).

[29] Tallur, S., Sridaran, S., Bhave, S. A. \& Carmon, T. Phase noise modeling of optomechanical oscillators arXiv: 1006.3805 (2010).

[30] Thompson, J. D. et al. Strong dispersive coupling of a high-finesse cavity to a micromechanical membrane. $\mathrm{Na}$ ture 452, 72-75 (2008). 